Available online at www.eccomasproceedia.org

Eccomas Proceedia EUROGEN (2021) 155-170

ECCOMAS

Proceedia
EUROGEN 2021

$14^{\text {th }}$ ECCOMAS Thematic Conference on Evolutionary and Deterministic Methods for Design, Optimization and Control

N. Gauger, K. Giannakoglou, M. Papadrakakis, J. Periaux (eds.) Streamed from Athens, Greece, 28-30 June 2021

\title{
EFFICIENT AND ACCURATE MODELING OF NON-PRISMATIC BEAMLIKE STRUCTURES
}

\author{
G. Migliaccio ${ }^{1}$ \\ ${ }^{1}$ Civil and Industrial Engineering, University of Pisa, \\ National Group of Mathematical Physics, Pisa \\ Largo Lucio Lazzarino 2, 56122, Pisa, Italy \\ e-mail: giovanni.migliaccio.it@gmail.com
}

\begin{abstract}
Many complex engineering structures, e.g. components of helicopters, wind turbines, aircraft wings and propellers, are beamlike and non-prismatic. Structures of this kind may be tapered, pre-twisted, and even curved in their unstressed state, and undergo large displacements and $3 D$ cross-sectional warping. Their mechanical modeling can be addressed via non-prismatic beam elements providing the appropriate compromise between computational efficiency and accuracy. Over the years many models have been proposed for beamlike structures, but general non-prismatic cases still require investigation. Formulas valid for prismatic beams, for example, generally provide incorrect results in non-prismatic cases, as the variation in the dimensions and orientation of the transverse cross-sections produce non-trivial stress distributions absent in prismatic beams. A model suitable for the aforementioned non-prismatic elements should properly describe their shape, explicitly consider the effects of their geometric design features on their stress and strain fields, account for large displacements, and provide the known results of prismatic cases. We propose a physical-mathematical model that accounts for all such requirements. The non-prismatic beam is seen as a collection of plane figures (the transverse cross-sections) attached at a 3D curve (the beam's centre-line). The centre-line's points may undergo large displacements. The transverse cross-sections are fully deformable and may undergo warpings in and out of plane. Assuming small warping and strain fields, a variational approach provides the field equations. The model obtained enables evaluating even analytically the effects of geometric parameters (such as taper) on the stress and strain fields. Numerical examples and comparisons with the results of nonlinear 3D-FEM analyses confirm the effectiveness of the proposed modeling approach.
\end{abstract}

Keywords: Non-prismatic beams, tapered cross-sections, large displacements. 


\section{INTRODUCTION}

Ongoing efforts to predict the mechanical behavior of non-prismatic beamlike structures, by using increasingly accurate and computationally efficient models, are aimed at optimizing their performance and cost. At the same time, tackling complex engineering problems continuously leads to develop theories to meet ever more stringent requirements on the predictive models. For example, several theories have been developed over the years for helicopter blades with pre-twist, a geometric feature which induces several couplings in their mechanical response: for pre-twisted beams bending is always three-dimensional, and tension may be significantly coupled to torsion [1-6]. Wind turbine blades are another interesting example of pre-twisted structures. Moreover, they are also characterized by important spanwise variations in the dimensions of their transverse cross-sections, that is, they are also tapered [7-8]. Their shape alone makes predicting their mechanical behavior a very challenging task. Not to speak of the large displacements they may undergo, which further complicate the study and derivation of analytical closed-form formulas for engineering design purposes.

Generally speaking, structural analyses show that the models and formulas commonly used for prismatic beams provide incorrect results in non-prismatic cases, as the variations in the dimensions and orientation of the transverse cross-sections produce non-trivial stress distributions absent in prismatic beams [9-10]. To date several models have been proposed for beamlike bodies, ranging from linear theories [11-13] to geometrically exact and asymptotic approaches [14-20]. However, investigations are still required to develop rigorous yet application-oriented models for three-dimensional non-prismatic beams, which explicitly account for important geometric features (such as taper), account for large displacements, directly furnish the $3 \mathrm{D}$ stress and strain fields, and provide known results in prismatic cases.

This paper presents a physical-mathematical model that meets all such requirements for non-prismatic beams having fully deformable cross-sections and undergoing large displacements and small strains. The general model is introduced in section 2. Analytical results for bi-tapered and pre-twisted beams are presented in section 3. Numerical examples and comparisons with the results of nonlinear 3D-FEM analyses are finally shown in section 4.

\section{MECHANICAL MODEL}

In this section we introduce the main ingredients of our model, further details of which can be found in [21]. Then, we present tapered beams for which we can provide analytical formulas for evaluating their stress and strain fields (section 3) and, subsequently, provide comparisons with the results of nonlinear 3D-FEM simulations (section 4).

\subsection{Geometry and strain measures}

The beam is seen as a collection of plane figures (transverse cross-sections) attached at a 3D curve (beam's centre-line). The cross-sections are fully deformable and may undergo displacements in and out of plane (all called warping displacements). The displacement of each cross-sectional point from the reference to the current state consists of a rigid part (similarly to the beam theories with rigid cross-sections) onto which the aforementioned warping motion is superposed. Figure 1 shows a schematic of the beam's reference and current states.

Two local triads of orthogonal unit vectors are introduced in Figure 1. The first, $b_{i}$, in the reference state, with $b_{1}$ tangent to the centre-line, depends on the reference arc-length s, i.e. $b_{i}=b_{i}(s)$. The second, $a_{i}$, is an image of $b_{i}$ in the current state and depends on the arc-length $s$ and time t, i.e. $a_{i}=a_{i}(s, t)$. A third triad, $c_{i}$, pertains to a fixed Cartesian reference frame. 


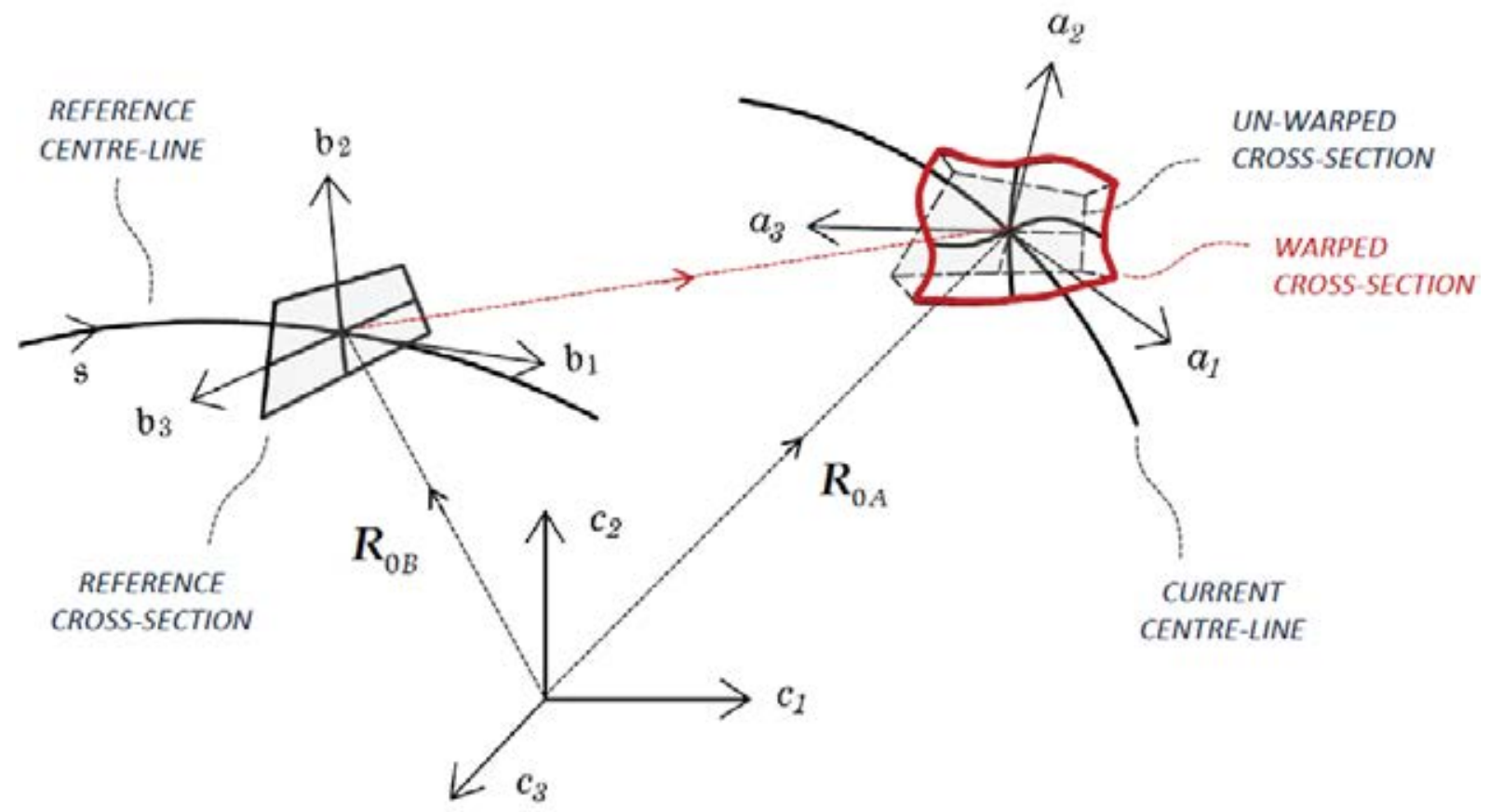

Figure 1: Schematic of current and reference states - centre-lines, cross-sections, local triads

Two mapping functions, $\mathrm{R}_{\mathrm{A}}$ and $\mathrm{R}_{\mathrm{B}}$, identify the positions of the beam's points in their current and reference states, respectively. The reference mapping function is

$$
R_{B}\left(z_{i}\right)=R_{0 B}\left(z_{1}\right)+x_{\alpha}\left(z_{i}\right) b_{\alpha}\left(z_{1}\right)
$$

where $\mathrm{R}_{0 в}$ provides the position of the reference center-line relative to triad $c_{i}, x_{\alpha}$ identify the position of the cross-section's points relative to such center-line, and $z_{i}$ are three mathematical variables, independent of time, with $\mathrm{z}_{1}=\mathrm{s}$, and $\mathrm{z}_{\alpha}$ belonging to a bi-dimensional domain used to map the position, $x_{\alpha}$, of the cross-section's points. Specifically, the spanwise variation of the shape of the transverse cross-sections is modeled via the map

$$
x_{i}=\Lambda_{i j} z_{j}
$$

where for the considered bi-tapered, pre-twisted beams $\Lambda_{11}=1, \Lambda_{22}=\Lambda_{2}\left(\mathrm{z}_{1}\right), \Lambda_{33}=\Lambda_{3}\left(\mathrm{z}_{1}\right)$, and the others $\Lambda_{\mathrm{ij}}$ are zero. Throughout this paper, Greek indices range from 2 to 3 , Latin indices take values from 1 to 3 , and repeated indices are summed over their range.

The current mapping function is defined, similarly to the reference one, as follows

$$
R_{A}\left(z_{i}, t\right)=R_{0 A}\left(z_{1}, t\right)+x_{\alpha}\left(z_{i}\right) a_{\alpha}\left(z_{1}, t\right)+w_{k}\left(z_{i}, t\right) a_{k}\left(z_{1}, t\right)
$$

where $\mathrm{R}_{0 \mathrm{~A}}$ denotes the position of the center-line's points in the current state, while $\mathrm{w}_{\mathrm{k}}$ are the components of the warping displacement with respect to triad $\mathrm{a}_{\mathrm{k}}$.

We now introduce vector and tensor fields useful to describe the motion of our beam, starting with the vector field $\mathrm{k}$, associated to the change in the beam's curvature between the current and reference states, given by

$$
k=T^{T} k_{A}-k_{B}
$$

where the proper orthogonal tensor field $T=a_{i} \otimes b_{i}$ describes the relative orientation between triads $a_{i}$ and $b_{i}, \otimes$ being the usual tensor (or dyadic) product, while the vector fields $k_{A}$ and 
$\mathrm{k}_{\mathrm{B}}$ are such that $a_{i}^{\prime}=k_{\mathrm{A}} \wedge a_{i}$ and $b_{i}^{\prime}=k_{B} \wedge b_{i}, \wedge$ being the usual cross-product and apex-prime denoting the derivative with respect to $\mathrm{s}$ (further details are in [21]).

Along with $\mathrm{k}$, we introduce another vector field, $\gamma$, which is related to the difference between the center-line tangent vectors of the current and reference states, as follows

$$
\gamma=T^{T} R_{0 A}^{\prime}-R_{0 B}^{\prime}
$$

Note that $\gamma$ and $\mathrm{k}$, which vanish for rigid motions and are invariant under superposed rigid motion [20], are referred to here as 1D strain measures. The Green-Lagrange strain tensor E is instead referred to here as 3D strain measure and is written in a form based on the assumption of small strain and warping fields considered in this work. In particular, we assume that the characteristic dimension of the transverse cross-sections, $h$, is much smaller than the reference length, $L$, of the centre-line (i.e. the beam is slender); the beam's curvatures are much smaller than $1 / h$; the warping fields, $w_{k}$, are considered small in the sense that their maximum order of magnitude is $h \varepsilon(\varepsilon<<1$ being a non-dimensional parameter), while the order of their derivative with respect to $z_{1}$ is at most $\varepsilon h / L$. In general, all components of the strain measures are considered small in the sense their order of magnitude is at most $\varepsilon$. For the considered beam, the strain tensor $\mathrm{E}$ is written (as in [9]) in the form

$$
E \simeq \frac{T^{T} H+H^{T} T}{2}-I
$$

where $\mathrm{H}$ is the gradient of transformation between the reference and current states

$$
H=\frac{\partial R_{A}}{\partial R_{B}}
$$

\subsection{Stress measures and balance equations}

The stress fields in our beam are determined supposing it to be elastic. For small strains the second Piola-Kirchhoff stress tensor S is linearly related to the strain tensor E, as follows

$$
S=\mathbb{C} E
$$

where $\mathbb{C}$ is the classical elasticity tensor [22], which characterizes the beam's material behavior (e.g. isotropic or not). For completeness' sake, we also introduce the first Piola-Kirchhoff stress tensor $\mathrm{P}$ and Cauchy stress tensor $\mathrm{C}$, which for our beam are $\mathrm{P}=\mathrm{TS}$ and $\mathrm{C}=\mathrm{TST}^{\mathrm{T}}$ (as in [9]). By using the stress tensor $\mathrm{P}$, cross-sectional stress resultants are defined in terms of two vector fields, $\mathrm{F}$ (force) and $\mathrm{M}$ (moment), as follows

$$
\begin{aligned}
& F=\int_{\Sigma} P_{i 1} a_{i} \\
& M=\int_{\Sigma} x_{\alpha} P_{i 1} a_{\alpha} \wedge a_{i}
\end{aligned}
$$

where $\Sigma$ is the cross-sectional domain and $P_{i j}=P \cdot a_{i} \otimes b_{j}$.

We now exploit the principle of expended power to derive balance equations for our beamlike body, which is three-dimensional and hyper-elastic [22]. To this end, its interactions with the external environment are quantified, for each velocity field attainable by the body, via the following functional, $\Pi_{e}$, called the external power

$$
\Pi_{e}=\int_{\partial V} p \cdot v+\int_{V} b \cdot v
$$


In (10), b are body loads per unit body's reference volume $\mathrm{V}, \mathrm{p}$ are surface tractions per unit area of the reference boundary $\partial \mathrm{V}$, and $\mathrm{v}$ is the referential description of the time rate of the current position of the body's points, given by

$$
v=v_{0}+\omega \wedge x_{\alpha} a_{\alpha}+w^{*}
$$

where $\mathrm{w}^{\bullet}$ is the time rate of the warping displacement. Interactions among different parts of the body are instead quantified via the functional $\Pi_{i}$, called the internal power

$$
\Pi_{i}=\frac{d}{d t} \int_{V} \Phi
$$

where $\Phi$ (body's energy density) is half the scalar product of tensors $\mathrm{S}$ and $\mathrm{E}$, i.e. $2 \Phi=\mathrm{S} \cdot \mathrm{E}$.

According to the principle of expended power, for any velocity field attainable by the body, its interactions with the external environment and among its parts are such that at any value of the evolution parameter $t$ the total power vanishes (i.e. $\Pi_{\mathrm{e}}=\Pi_{\mathrm{i}}$ ). The exploitation of such principle is a usual technique in continuum mechanics to obtain balance equations in terms of the problem's unknowns (see, e.g., [20-22]). In our case, it enables writing balance equations for the stress resultants, $\mathrm{F}$ and $\mathrm{M}$, in the form

$$
\begin{aligned}
& F^{\prime}+f=0 \\
& M^{\prime}+R_{0 A}^{\prime} \wedge F+m=0
\end{aligned}
$$

where $\mathrm{f}$ and $\mathrm{m}$ are the resultants of the body and contact actions per unit length of reference centre-line. The same principle also enables writing balance equations to determine the warping fields $\mathrm{w}_{\mathrm{k}}$. In particular, in the case the body loads and surface tractions on the beam's lateral surface are neglected in calculating the warping fields, or vanish, it is possible to reduce the determination of the warping fields to those that verify the variational condition

$$
\delta \int_{V} \Phi=0
$$

where $\delta$ denotes the variation of the energy function with respect to the warping fields. Note that warping fields satisfying condition (14) can be obtained numerically or, in particular cases, analytically, as solutions of the corresponding Euler-Lagrange equations [23].

So far we have introduced the main ingredients of our modeling approach. Specifically, we have sketched the body's shape, which is three-dimensional and beamlike, and have defined the strain measures, stress measures, and balance equations we use to describe its mechanical behavior. By using such ingredients, the resolution of the three-dimensional nonlinear elasticity problem is now reduced to the solution of two main problems: the first governs the cross-sectional warping motion and it's strong formulation can be given in terms of partial differential equations (PDEs) defined over a reference bi-dimensional domain (as is discussed in the next section). The second problem governs the centre-line motion and can be expressed in terms of a set of non-linear ordinary differential equations (ODEs) defined over a reference line (as in [9], for instance). It is worth noting that this approach reduces the computational effort and produces accurate results compared to nonlinear 3D-FEM approaches (as is shown also in the following). Moreover, the results obtained with our model enable evaluating even analytically the effects of important geometric features, such as the cross-sectional taper, on the stress and strain fields in all points of the considered beamlike bodies. The analytical results obtained in this work are presented in the next section 3 and, subsequently, are compared to the results of nonlinear 3D-FEM approaches (section 4). 


\section{ANALITYCAL RESULTS}

In order to determine the stress and strain fields in our beam we need to exploit the variational condition (14). In particular, considering the corresponding Euler-Lagrange equations, in which we keep the terms up to the order $\varepsilon h / L$, we can obtain a set of partial differential equations (PDEs) with Neumann-type boundary conditions the solution of which enables determining the components of tensor E. In doing this, hereafter we choose the current local triads to be tangent to the center-line and focus on the case of bi-tapered beams the material properties of which are described in terms of just two material constants (i.e., Young's modulus $Y$, and Poisson's ratio $v$ ). The effects of other geometric features (e.g. centre-line curvature), as well as those related to the material non-homogeneity and anisotropy, will be addressed in subsequent works.

Proceeding as in [21], the components $\mathrm{E}_{11}, \mathrm{E}_{21}$, and $\mathrm{E}_{31}$ of $\mathrm{E}$, related to the out-of-plane deformation of the transverse cross-sections, can be written in the form

$$
\begin{aligned}
& E_{11}=k_{2} x_{3}-k_{3} x_{2}+\gamma_{1}+e_{1,1}+k_{B 1}\left(x_{3} e_{1,2}-x_{2} e_{1,3}\right) \\
& 2 E_{21}=e_{1,2}-k_{1} x_{3}+e_{2}+2(1+v)\left(k_{2} x_{3}-k_{3} x_{2}+\gamma_{1}\right)\left(\Lambda_{2}^{-1} \Lambda_{2}^{\prime} x_{2}-k_{B 1} x_{3}\right) \\
& 2 E_{31}=e_{1,3}+k_{1} x_{2}+e_{3}+2(1+v)\left(k_{2} x_{3}-k_{3} x_{2}+\gamma_{1}\right)\left(\Lambda_{3}^{-1} \Lambda_{3}^{\prime} x_{3}+k_{B 1} x_{2}\right)
\end{aligned}
$$

where $E_{i j}=E \cdot b_{i} \otimes b_{j}$, subscript-comma denotes the derivative with respect to $\mathrm{x}_{\mathrm{i}}$, and the scalar fields $\mathrm{e}_{1}, \mathrm{e}_{2}, \mathrm{e}_{3}$ are solutions of the following PDEs problems

$$
\begin{aligned}
& e_{1,22}+e_{1,33}=0 \quad \text { in } \Sigma \\
& \left(e_{1,2}-k_{1} x_{3}\right) n_{2}+\left(e_{1,3}+k_{1} x_{2}\right) n_{3}=0 \quad \text { on } \partial \Sigma \\
& e_{2,2}+e_{3,3}=d_{2} x_{2}+d_{3} x_{3} \quad \text { in } \Sigma \\
& e_{3,2}-e_{2,3}=g_{2} x_{2}+g_{3} x_{3}+g_{1} \quad \text { in } \Sigma \\
& e_{2} n_{2}+e_{3} n_{3}=0 \quad \text { on } \partial \Sigma
\end{aligned}
$$

In (16), $\Sigma$ and $\partial \Sigma$ are the cross-sectional domain and its boundary, $\mathrm{n}_{\alpha}$ are the components of the outward unit normal vectors on $\partial \Sigma$, and coefficients $\mathrm{d}_{\alpha}$ and $\mathrm{g}_{\mathrm{k}}$ are given by

$$
\begin{aligned}
& d_{2}=+2(1+v) k_{3}^{\prime}+2(1+v)\left(\Lambda_{3}^{-1} \Lambda_{3}^{\prime}+2 \Lambda_{2}^{-1} \Lambda_{2}^{\prime}\right) k_{3} \\
& d_{3}=-2(1+v) k_{2}^{\prime}-2(1+v)\left(\Lambda_{2}^{-1} \Lambda_{2}^{\prime}+2 \Lambda_{3}^{-1} \Lambda_{3}^{\prime}\right) k_{2} \\
& g_{1}=2 k_{B 1}(2+2 v) \gamma_{1} \\
& g_{2}=-2 v k_{2}^{\prime}-2(1+v) \Lambda_{2}^{-1} \Lambda_{2}^{\prime} k_{2}-2 k_{B 1}(3+2 v) k_{3} \\
& g_{3}=-2 v k_{3}^{\prime}-2(1+v) \Lambda_{3}^{-1} \Lambda_{3}^{\prime} k_{3}+2 k_{B 1}(3+2 v) k_{2}
\end{aligned}
$$

It is worth noting that the PDEs equations (16) formally resemble those for flexure and torsion of a Saint-Venant's cylinder. Unfortunately, PDEs problems of this kind can be solved in closed-form only for a few cases, but this is not surprising (this happens even in the linear theory of prismatic beams [11-13]). However, they can always be solved with the aid of numerical methods for all other cases as well. Regarding our PDEs problem (16)-(17), we moreover note that its solution can generally be expressed as linear combinations of the 1D strain measures, $\gamma_{1}$ and $\mathrm{k}_{\mathrm{i}}$, and their $s$-derivative, and explicitly depends on the beam's reference shape through the shape of the cross-sectional domain $\Sigma$ and some application-oriented functions, e.g. the taper coefficients $\Lambda_{\alpha}$ and pre-twist coefficient $\mathrm{k}_{\mathrm{B} 1}$. 
The results discussed right now are particularly interesting because the nature of our PDEs problem allows considering even separately the effects of the different $1 \mathrm{D}$ strain measures and geometric parameters $\left(\Lambda_{\alpha}\right.$ and $\left.\mathrm{k}_{\mathrm{B} 1}\right)$ in the determination of the scalar fields $\mathrm{e}_{1}, \mathrm{e}_{2}, \mathrm{e}_{3}$, which are necessary for calculating the strain fields (15), stress fields (8), and stress resultants (9).

Note that the approach used can also provide expressions for the strain fields $E_{22}, E_{33}, E_{23}$, related to the cross-sectional in-plane deformation, plus the relevant PDEs problem. However, we do not provide details about this in the present paper, but focus on the scalar fields $\mathrm{e}_{1}, \mathrm{e}_{2}$, and $\mathrm{e}_{3}$, and discuss some cases in which they can be obtained in closed-form.

\subsection{Tapered beam with circular solid cross-sections}

As anticipated in the foregoing, the PDEs problem (16)-(17) admits closed-form analytical solutions in some cases. This holds, for instance, for circularly cross-sectioned tapered beams with taper coefficients $\Lambda_{2}=\Lambda_{3}=\Lambda$ and pre-twist $\mathrm{k}_{\mathrm{B} 1}=0$. In such case, in fact, equations (16)-(17) are satisfied by $\mathrm{e}_{1}=0$ and the expressions of $\mathrm{e}_{2}$ and $\mathrm{e}_{3}$ that follow

$$
\begin{aligned}
& e_{2}=p_{1} k_{3}^{\prime}+p_{2} k_{2}^{\prime}+2(1+v) \Lambda^{-1} \Lambda^{\prime}\left[\left(x_{2}^{2}-R^{2}\right) k_{3}-x_{2} x_{3} k_{2}\right] \\
& e_{3}=q_{1} k_{2}^{\prime}+q_{2} k_{3}^{\prime}+2(1+v) \Lambda^{-1} \Lambda^{\prime}\left[\left(R^{2}-x_{3}^{2}\right) k_{2}+x_{3} x_{2} k_{3}\right]
\end{aligned}
$$

where $\mathrm{R}$ is the radius of the transverse cross-section (which depends on $\mathrm{s}$, as it is scaled from the root to the tip of the beam according to the taper function $\Lambda$ ), and

$$
\begin{aligned}
& 2 p_{1}=(1-2 v) x_{3}^{2}+(3+2 v)\left(x_{2}^{2}-R^{2}\right) \\
& p_{2}=-(1+2 v) x_{2} x_{3} \\
& 2 q_{1}=-(1-2 v) x_{2}^{2}-(3+2 v)\left(x_{3}^{2}-R^{2}\right) \\
& q_{2}=(1+2 v) x_{3} x_{2}
\end{aligned}
$$

Given $\mathrm{e}_{1}, \mathrm{e}_{2}, \mathrm{e}_{3}$, we can also write closed-form expressions for the strain fields $\mathrm{E}_{11}, \mathrm{E}_{21}, \mathrm{E}_{31}$ by combining (18)-(19) with (15). In particular, such strain fields can be expressed in terms of linear combinations of the 1D strain measures and their s-derivatives, as follows

$$
\begin{aligned}
& E_{11}=k_{2} x_{3}-k_{3} x_{2}+\gamma_{1} \\
& 2 E_{21}=-k_{1} x_{3}+p_{1} k_{3}^{\prime}+p_{2} k_{2}^{\prime}+p_{3} k_{3}+p_{4} \gamma_{1} \\
& 2 E_{31}=+k_{1} x_{2}+q_{1} k_{2}^{\prime}+q_{2} k_{3}^{\prime}+q_{3} k_{2}+q_{4} \gamma_{1}
\end{aligned}
$$

where the additional coefficients, $\mathrm{p}_{3}, \mathrm{p}_{4}, \mathrm{q}_{3}$, and $\mathrm{q}_{4}$, of the linear combinations are given by

$$
\begin{aligned}
& p_{3}=-2(1+v) \Lambda^{-1} \Lambda^{\prime} R^{2} \\
& p_{4}=2(1+v) \Lambda^{-1} \Lambda^{\prime} x_{2} \\
& q_{3}=2(1+v) \Lambda^{-1} \Lambda^{\prime} R^{2} \\
& q_{4}=2(1+v) \Lambda^{-1} \Lambda^{\prime} x_{3}
\end{aligned}
$$

Note that such solution (18)-(21), which is valid for the circularly cross-sectioned tapered beams considered here, undergoing large displacements and small strains, reduces exactly to that of the Saint-Venant's linear theory if $\Lambda^{\prime}$ vanishes (prismatic case) and both the beam's displacements and strains are small. In such case, in fact, functions $\mathrm{p}_{3}, \mathrm{p}_{4}, \mathrm{q}_{3}, \mathrm{q}_{4}$ vanish, while functions $\mathrm{p}_{1}, \mathrm{p}_{2}, \mathrm{q}_{1}, \mathrm{q}_{2}$, which are proportional to $\mathrm{k}_{2}^{\prime}$ and $\mathrm{k}_{3}^{\prime}$ (i.e. the derivatives of the bending 
curvatures), turn out to be proportional to the cross-sectional shear forces in the beam's reference state, as in the works of Timoshenko [12] and Sokolnikoff [13].

It is worth noting that similar but generally more complex expressions for $E_{11}, E_{21}, E_{31}$ can be obtained for other non-prismatic beams as well (see, e.g., [6,21]). However, we do not discuss here all cases in which we can obtain closed-form solutions to problem (16)-(17), but we present another interesting example (in section 3.2) and then proceed to compare the results of our model with those of nonlinear 3D-FEM simulations to verify its effectiveness in terms of computational efficiency and accuracy (section 4).

Before doing this, we remark that regardless of the approach used (i.e. numerical or analytical) to solve our problem (16)-(17), the solution can always be expressed in terms of linear combinations of terms proportional to the 1D strain measures and their s-derivative, similarly to (18)-(20). The difference between the solution reported in this section and that of a generic bi-tapered, pre-twisted beam is represented by the expressions of the coefficients of the linear combinations (e.g. $\mathrm{p}_{1}-\mathrm{p}_{4}$ ), which are the functions of $\mathrm{x}_{\alpha}$ to be found. Such functions, however, have to be computed only once for a given cross-sectional shape. This fact, of course, contributes reducing the computational effort required to solve the nonlinear elasticity problem that governs the behavior of our three-dimensional beams, as is shown in section 4 via numerical examples and comparisons with the results of nonlinear 3D-FEM analyses.

\subsection{Tapered beam with circular hollow cross-sections}

Let us now consider a tapered beam similar to that of the previous section, but having hollow cross-sections. Specifically, here we are considering thin-walled cross-sections, characterized by small thickness-to-radius ratio, $\mathrm{t} / \mathrm{R}$. For example, we can refer to the case in Figure 2 , but without prescription on the shape of the taper function $\Lambda$ (which does not need to be a linear function for the derivation of the following formulas).
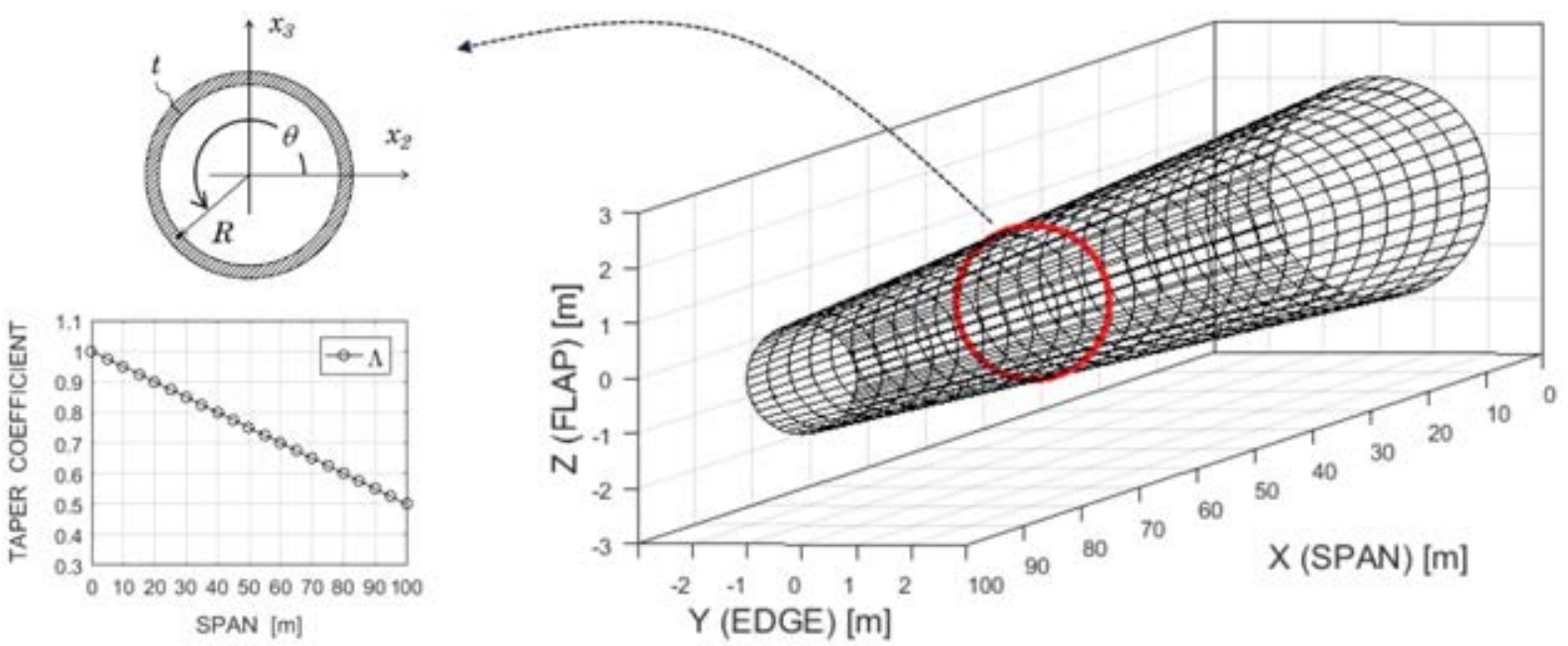

Figure 2: Tapered beam with circular hollow cross-sections (right) and its taper coefficient (left)

Regarding functions $\mathrm{e}_{1}, \mathrm{e}_{2}, \mathrm{e}_{3}$ for the present beam, a vanishing $\mathrm{e}_{1}$ still satisfies the PDEs problem (16)-(17), but the expressions of $\mathrm{e}_{2}, \mathrm{e}_{3}$ obtained for the circular solid cross-sections in section 3.1 do not satisfy all PDEs and boundary conditions (16)-(17) of the present case. For their determination, apart from using a numerical method, we can exploit, here, a simplified analytical approach (common for thin-walled beams) based on the assumption that the strain and stress fields may not vary too much through the cross-sectional thickness. 
By using such approach, we proceed to derive formulas for the strain fields (15) valid for the present beam subject to flexure and express such formulas as functions of the bending curvatures and their $s$-derivatives. To this end, it is convenient to consider the components $\mathrm{E}_{11}$, $E_{\theta 1}, E_{r 1}$ of $E$ (in place of $E_{11}, E_{21}, E_{31}$ ), $r$ and $\theta$ being the independent variables along the radial and tangential directions in the cross-section (see, e.g., Figure 2). Moreover, we also introduce the mean value $\mathrm{E}_{\theta}$, through the cross-sectional thickness $\mathrm{t}$, of the tangential shear strain $\mathrm{E}_{\theta 1}$, which is given by the following line-integral through the thickness

$$
E_{\theta}=\frac{1}{t} \int_{\text {tickness }}-E_{21} \sin \theta+E_{31} \cos \theta
$$

By exploiting (22), (15)-(16), and standard integration techniques based on Green's formulas, we can write $\mathrm{E}_{\theta}$ in the following form

$$
E_{\theta}=(1+v)\left(k_{3}^{\prime}+3 \Lambda^{-1} \Lambda^{\prime} k_{3}\right) R^{2} \sin \theta+(1+v)\left(k_{2}^{\prime}+3 \Lambda^{-1} \Lambda^{\prime} k_{2}\right) R^{2} \cos \theta
$$

The mean value $\mathrm{E}_{\mathrm{r}}$ of the radial shear strain $\mathrm{E}_{\mathrm{r} 1}$ is instead obtained by exploiting the condition of zero traction on the beam's lateral surface, which yields

$$
E_{r}=(1+v)\left(k_{2} \sin \theta-k_{3} \cos \theta\right) R^{2} \Lambda^{-1} \Lambda^{\prime}
$$

Now, the approximation relies on considering the local strain fields almost coincident with their mean value over the cross-sectional thickness, i.e. $E_{\theta 1} \simeq E_{\theta}$ and $E_{r 1} \simeq E_{r}$. Such assumption is expected to provide good results in terms of predictions of the cross-sectional strain and stress fields in thin-walled tapered beams, the cross-sectional thickness of which is small with respect to the cross-sectional diameter or radius (e.g. $\mathrm{t} / \mathrm{R} \simeq 0.1$ ).

The following examples provide comparisons with the results of nonlinear 3D-FEM simulations to verify the effectiveness of the modeling approach and formulas discussed so far.

\section{APPLICATION EXAMPLES}

In this section we shows the results obtainable by our model, which we have implemented in a numerical code written in Matlab language, referred to here as 3D-BLM. The results from 3D-BLM in terms of displacement, strain and stress fields are compared to those of nonlinear 3D-FEM analyses performed with Ansys, based on a fine mesh of solid tetrahedral elements with ten nodes and quadratic displacement behavior [24].

Two test cases are reported here. The first addresses a tapered beam with circular solid cross-sections, which may undergo large displacements while fixed at one end (the root) and loaded at the other (the tip) by a transverse force of progressively increasing magnitude. For such case we can exploit the analytical results presented in section 3.1. The second test case is similar to the first, but the transverse cross-sections are hollow. For such case we exploit the formulas presented in section 3.2.

\subsection{Test case 1 (solid cross-sections)}

The tapered beam considered here has a straight, $100 \mathrm{~m}$ long centre-line; its transverse cross-sections are circular, with a radius $\mathrm{R}=2 \mathrm{~m}$ at the root, which is linearly scaled toward the tip up to $30 \%$ of the root value. The material properties are given in terms of Young's modulus, 70GPa, and Poisson's ratio, 0.25. The beam is fixed at the root and loaded at the tip by a flapwise dead force, $F$, ranging from $100 \mathrm{kN}$ to $15000 \mathrm{kN}$ (as in Figure 3). The simulation results are summarized in the following. 
Figure 3 provides an overview of the beam's un-deformed state $(F=0)$, its deformed states obtained from 3D-BML for $F=5000 \mathrm{kN}$ and $F=10000 \mathrm{kN}$ (left), and the deformed state given by nonlinear 3D-FEM for $F=5000 \mathrm{kN}$ (right). Figures 4 and 5, instead, provide comparisons between 3D-BLM and 3D-FEM in terms of centre-line's displacements and simulation times for increasing $F$. As we can see, the simulation time with $3 \mathrm{D}-\mathrm{BLM}$ is much smaller than that required by nonlinear 3D-FEM, while the accuracy of results is always almost the same.

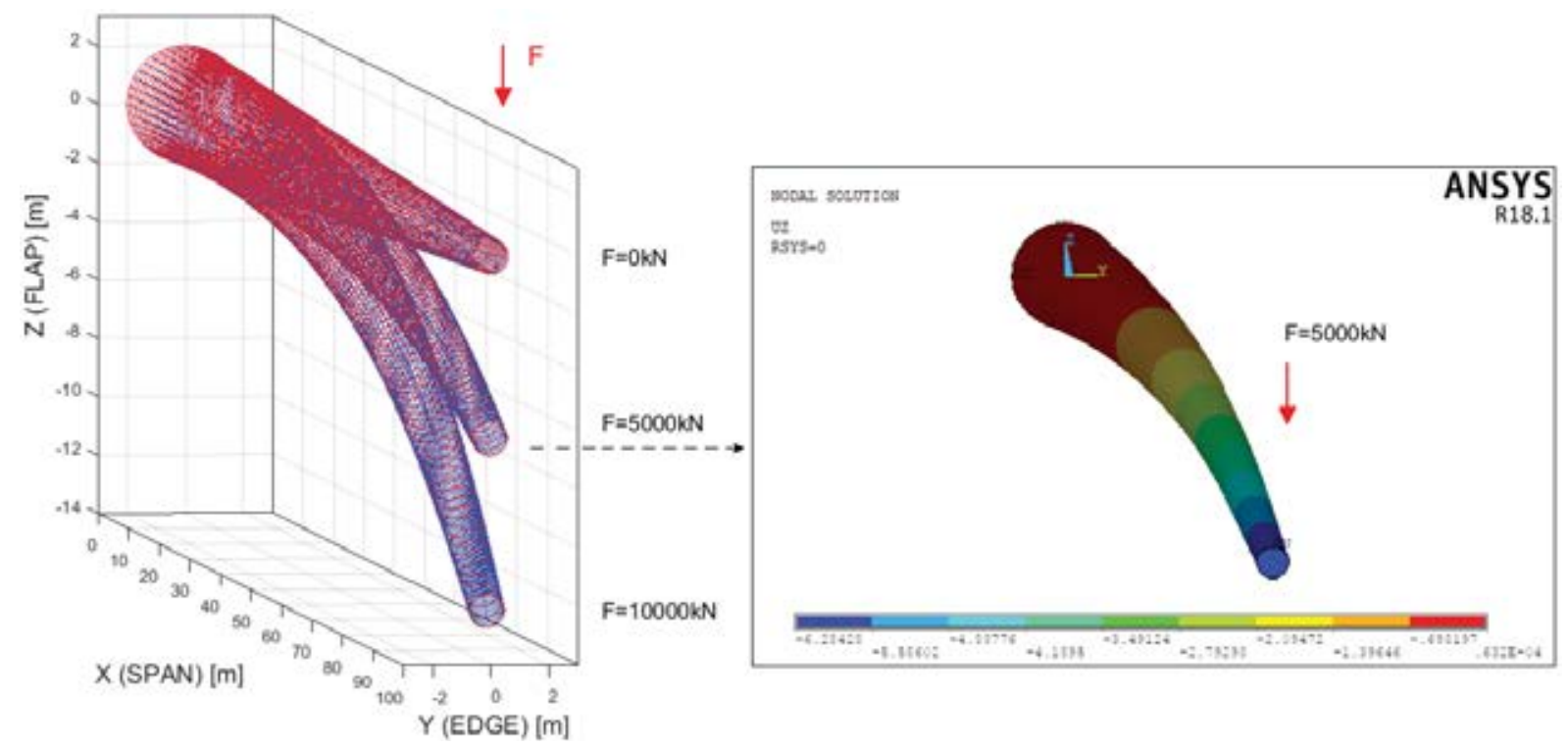

Figure 3: Beam's deflected shapes with 3D-BLM for increasing F (left) and 3D-FEM for F=5000kN (right)
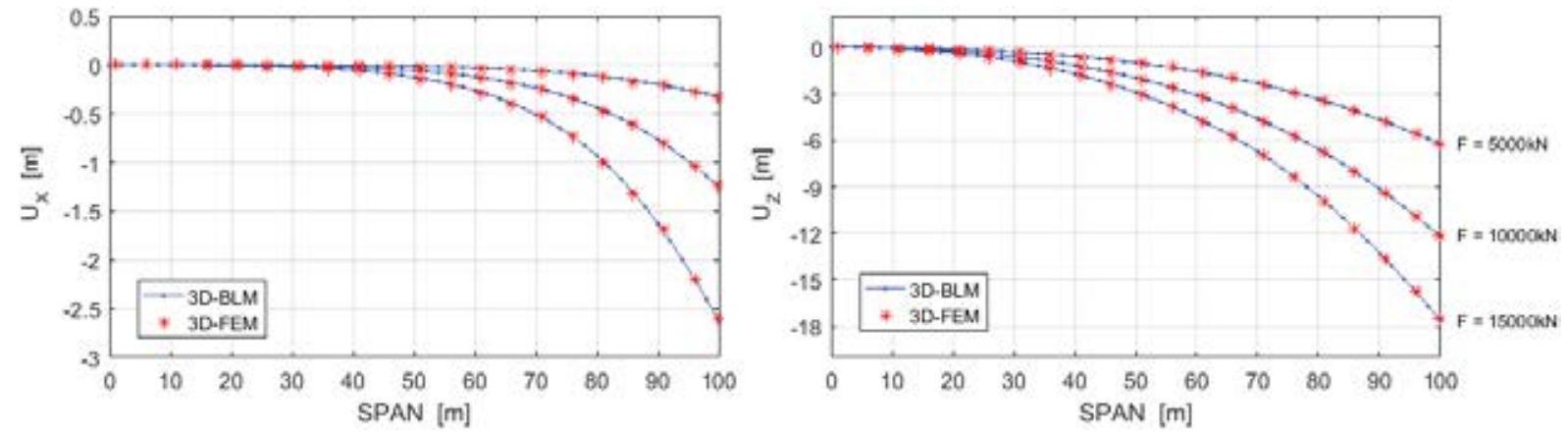

Figure 4: Comparing 3D-BLM and 3D-FEM in terms of centre-line's displacements along X (left) and Z (right)
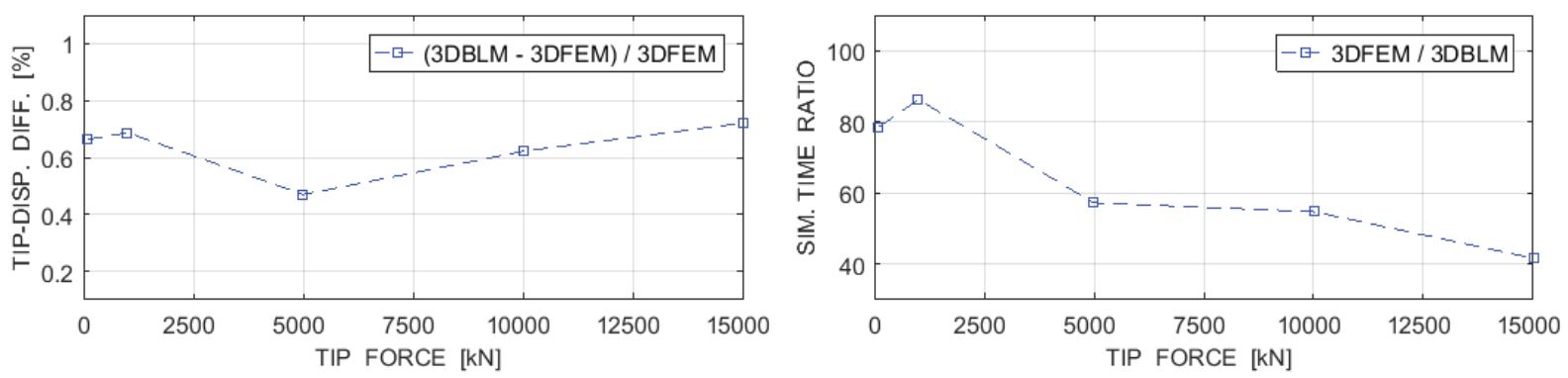

Figure 5: Comparing 3D-BLM and 3D-FEM in terms of tip-displacements (left) and simulation times (right) 
Apart from this, our model can also provide other useful information about the mechanical behavior of our beam, e.g. the rotation of its local triads, its curvature change and strain fields, as well as the corresponding stress fields and stress resultants.

Figure 6 , for example, reports the rotation $\theta_{\mathrm{Y}}$ of the local triads about $\mathrm{Y}$, along with the curvature change about $\mathrm{Y}\left(\mathrm{k}_{\mathrm{Y}}\right)$, for increasing $F$. It is worth noting that the spanwise variation of the bending curvature $\mathrm{k}_{\mathrm{Y}}$ is quite different from a linear function. However, this is expected for tapered beams (in spite of what would happen in a prismatic case under the same loading condition). This depends, in fact, on the spanwise variation of the cross-sectional bending stiffness which, in the present case, increases from the beam's tip to the root according to the increasing cross-sectional diameter.
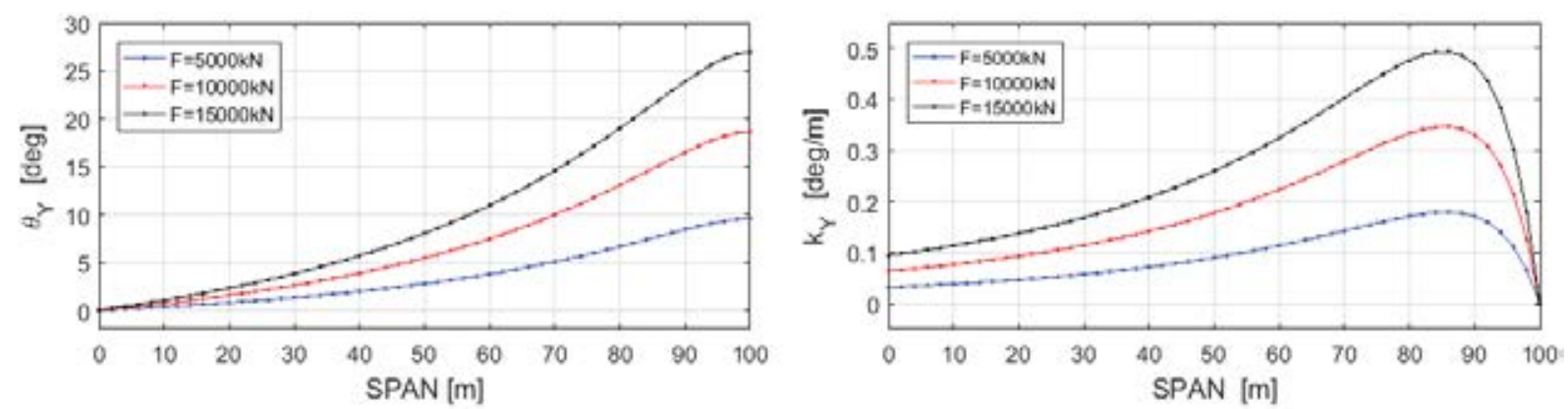

Figure 6: Local triads rotation $\theta_{\mathrm{Y}}$ (left) and bending curvature $\mathrm{k}_{\mathrm{Y}}$ (right) with 3D-BLM for increasing F

As anticipated in the foregoing, 3D-BLM can also directly furnish the stress fields in all points of the beam. Hereafter we compare the results obtained in terms of Cauchy stress fields from 3D-BLM with those given by nonlinear 3D-FEM simulations.

Specifically, Figures 7 and 8 show the stress fields $C_{X X}$ and $C_{Z X}$ obtained for $F=100 \mathrm{kN}$ at three reference cross-sections $(30 \%, 50 \%, 70 \%$ span). For completeness' sake, we also report the results obtained for a much larger value of $F$ (see Figures 9 and 10, $F=10000 \mathrm{kN}$ ). Similar results have moreover been obtained at other cross-sections and for other values of $F$.

Taking a look at the results obtained, we have observed that the normal stresses follow a Navier-like distribution in each cross-section (i.e. they are almost linear in $\mathrm{x}_{3}$ ), while the shear stress distributions are quite different from those predictable by the linear theory of prismatic beams. In fact, the transverse shear stresses at the cross-section's boundary do not generally vanish in non-prismatic beams, while they are always zero in the prismatic case. Also, they can change from cross-section to cross-section in non-prismatic elements, while their distribution and magnitude do not change spanwise in the prismatic case.
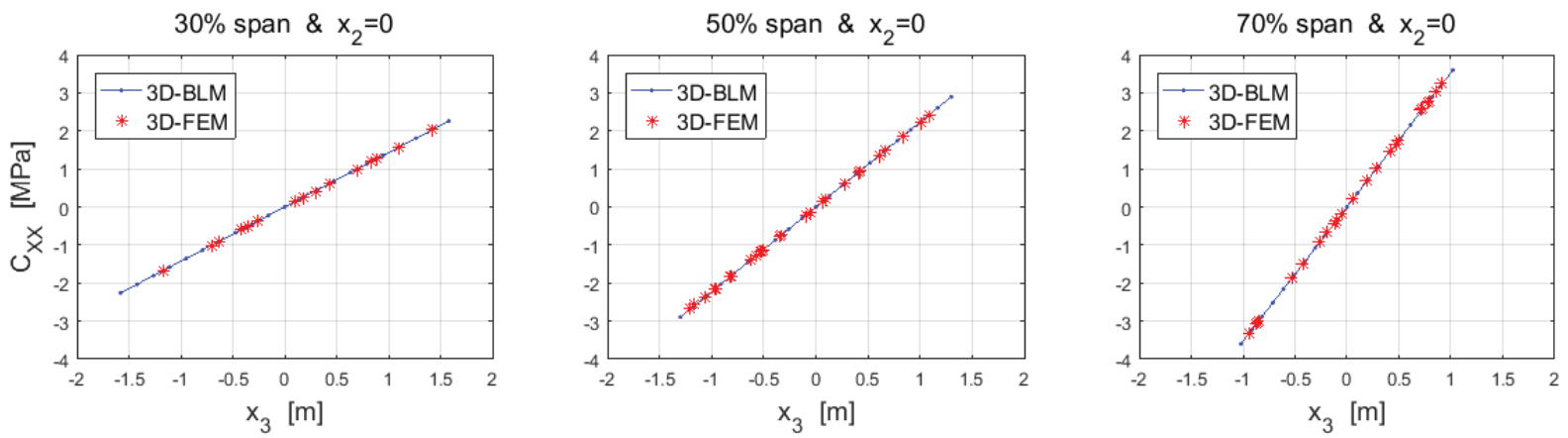

Figure 7: Stress field $\mathrm{C}_{\mathrm{XX}}$ at different cross-sections $(30 \%, 50 \%, 70 \%$ span) for $\mathrm{F}=100 \mathrm{kN}$ 

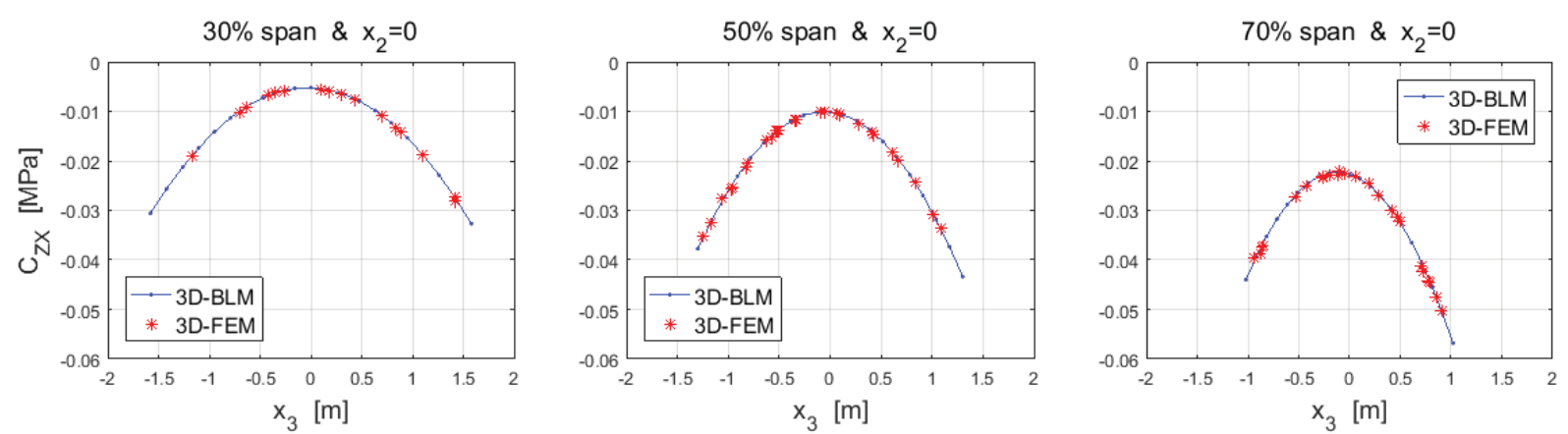

Figure 8: Stress field $\mathrm{C}_{\mathrm{ZX}}$ at different cross-sections $(30 \%, 50 \%, 70 \%$ span) for $\mathrm{F}=100 \mathrm{kN}$
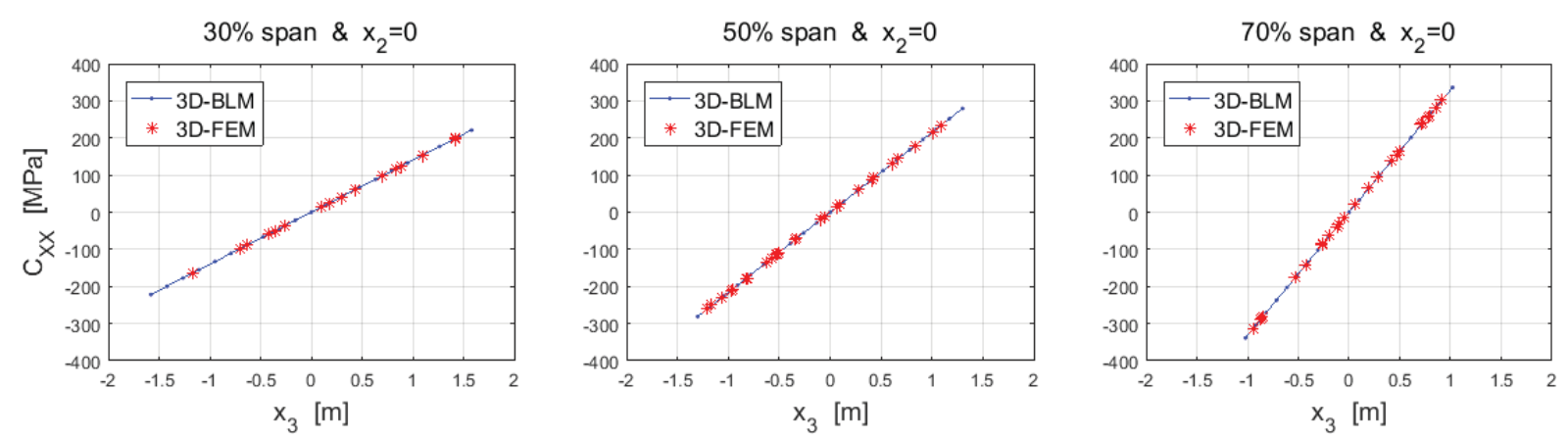

Figure 9: Stress field $\mathrm{C}_{\mathrm{XX}}$ at different cross-sections $(30 \%, 50 \%, 70 \%$ span) for $\mathrm{F}=10000 \mathrm{kN}$
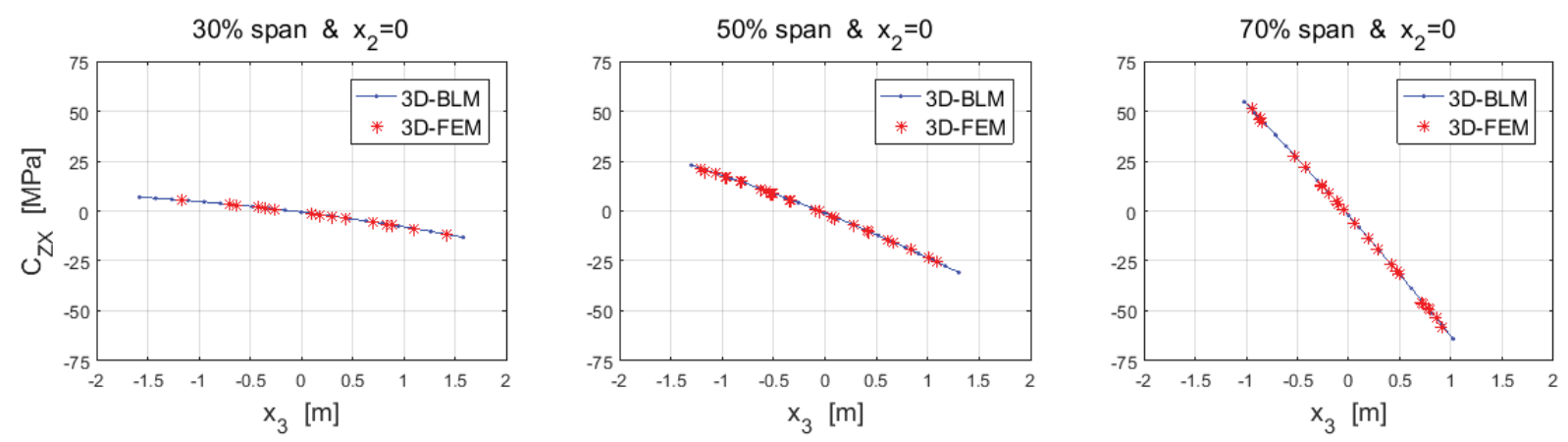

Figure 10: Stress field $\mathrm{C}_{\mathrm{ZX}}$ at different cross-sections $(30 \%, 50 \%, 70 \%$ span) for $\mathrm{F}=10000 \mathrm{kN}$

The results obtained so far confirm the effectiveness of the modeling approach introduced in the foregoing and the accuracy of the closed-form solution of section 3.1.

\subsection{Test case 2 (hollow cross-sections)}

The second test case considered here addresses a straight beam with $100 \mathrm{~m}$ long centre-line and circularly shaped hollow cross-sections, which are tapered from the root to the tip of the beam. The radius of the hollow root section is $R=2 \mathrm{~m}$, while its thickness is $t=0.2 \mathrm{~m}$. Such dimensions are linearly reduced toward the beam's tip as shown in Figure 2. The material properties are summarized by reference values of Young's modulus, 70GPa, and Poisson's ratio, 0.25 . The beam is fixed at the root and loaded at the tip by a flapwise dead force, $F$, ranging from $100 \mathrm{kN}$ to $10000 \mathrm{kN}$ (as in Figure 11). 


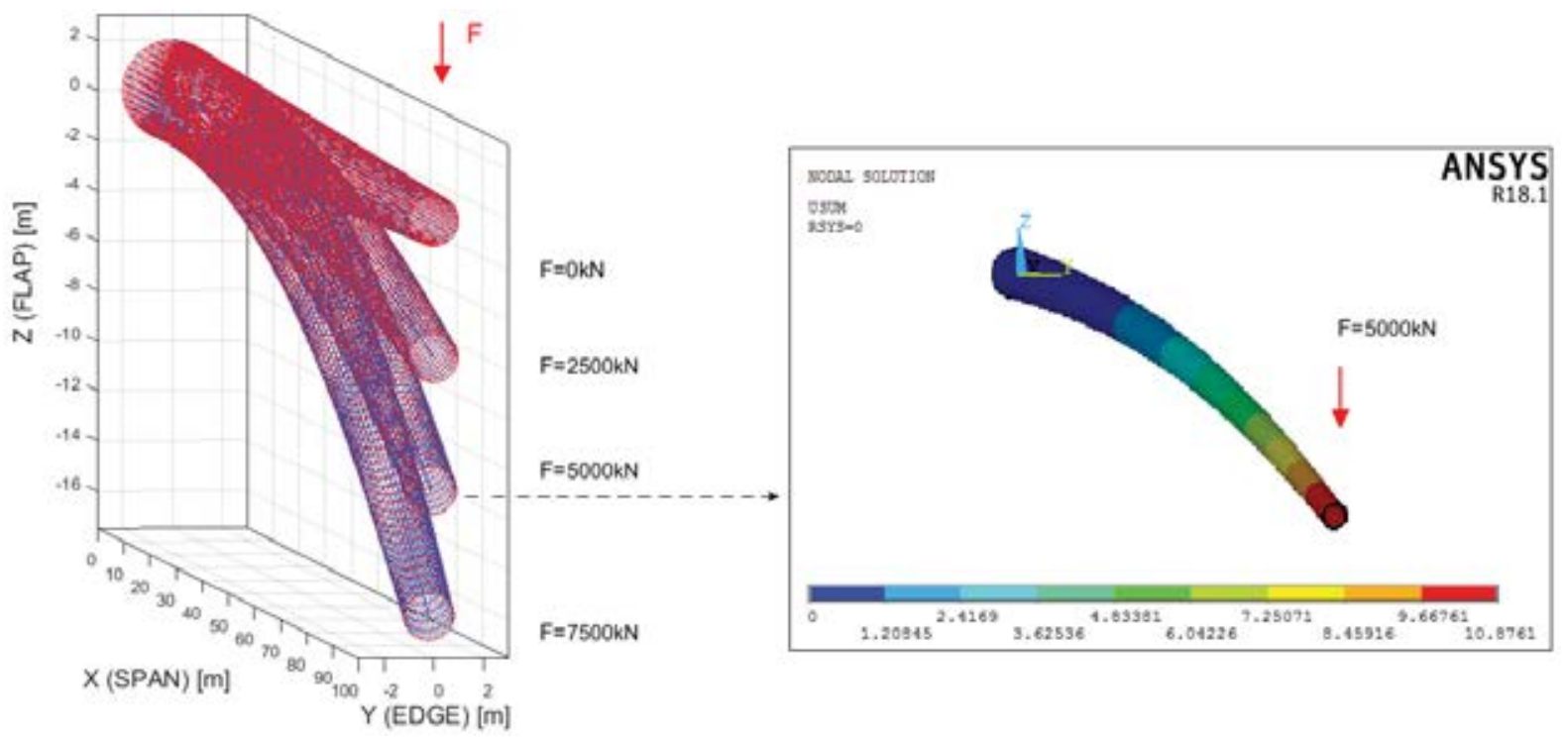

Figure 11: Beam's deflected shapes with 3D-BLM for increasing F (left) and 3D-FEM for F=5000kN (right)

Also in this case we can obtain the same sort of results shown in section 4.1. For example, Figure 11 reports the beam's deformed shapes obtained from 3D-BLM for different values of $F$, as well as the deformed shape given by $3 \mathrm{D}-\mathrm{FEM}$ for $F=5000 \mathrm{kN}$. Figure 12 provides comparisons between 3D-BLM and 3D-FEM in terms of tip-displacements and simulation times, which confirm the levels of computational efficiency and accuracy noticed in the previous test case (section 4.1). Figure 13 shows the displacements of the centre-line's points along $X$ and $\mathrm{Z}$ given by 3D-BLM (blue lines) and 3D-FEM (red marks) for increasing $F$. Finally, Figures 14 to 16 report the comparisons (between 3D-BLM and 3D-FEM) in terms of Cauchy stress fields. In particular, Figure 15 shows the normal stress $\mathrm{C}_{\mathrm{XX}}$ obtained for $F=5000 \mathrm{kN}$ at three cross-sections $(30 \%, 50 \%, 70 \%$ span), while the corresponding shear stresses along the tangential and radial directions, $\mathrm{C}_{\theta \mathrm{X}}$ and $\mathrm{C}_{\mathrm{RX}}$, are in Figures 16 and 17.

Regarding the stress fields, it is worth noting that also in this test case the normal stresses follow a Navier-like distribution in the transverse cross-sections, while the shear stress distributions are quite different from those observable in prismatic beams with circular hollow cross-sections. For example, the tangential shear stress distributions are observed to change spanwise (i.e. from the root section to the tip section), while they would be the same at every cross-section in the prismatic case. In addition, in the present case we also have radial shear stresses, which, instead, are absent in the prismatic case.
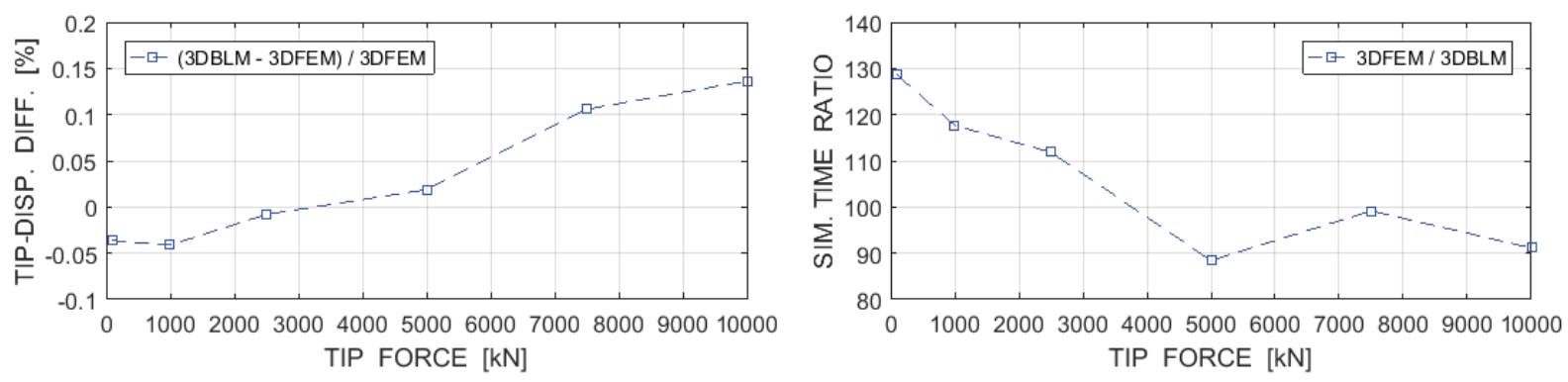

Figure 12: Comparing 3D-BLM and 3D-FEM in terms of tip-displacements (left) and simulation times (right) 

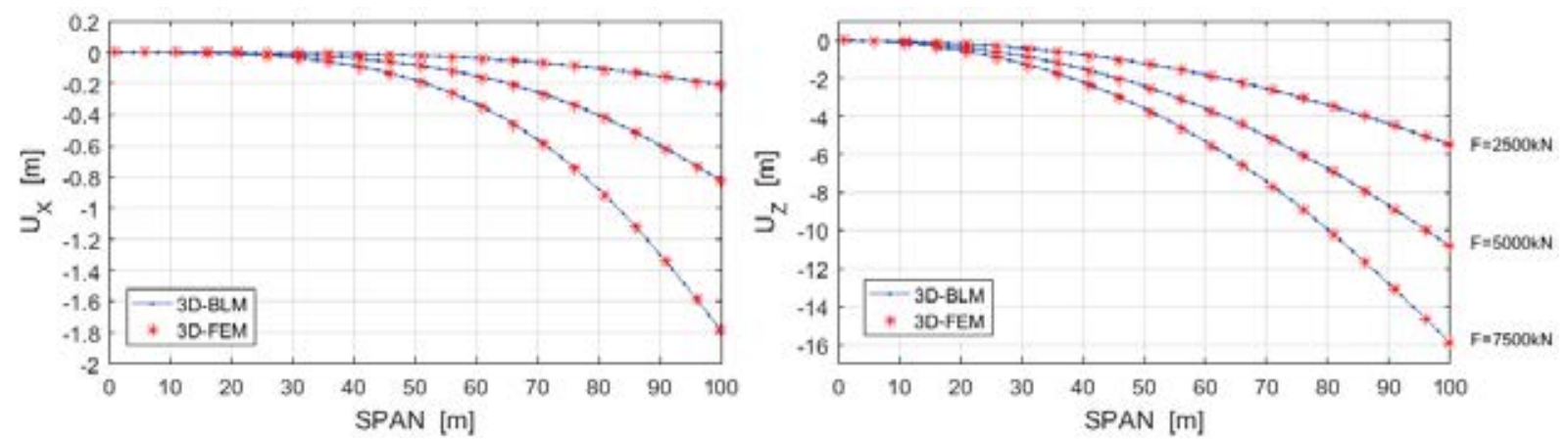

Figure 13: Comparing 3D-BLM and 3D-FEM in terms of displacements along X (left) and Z (right)
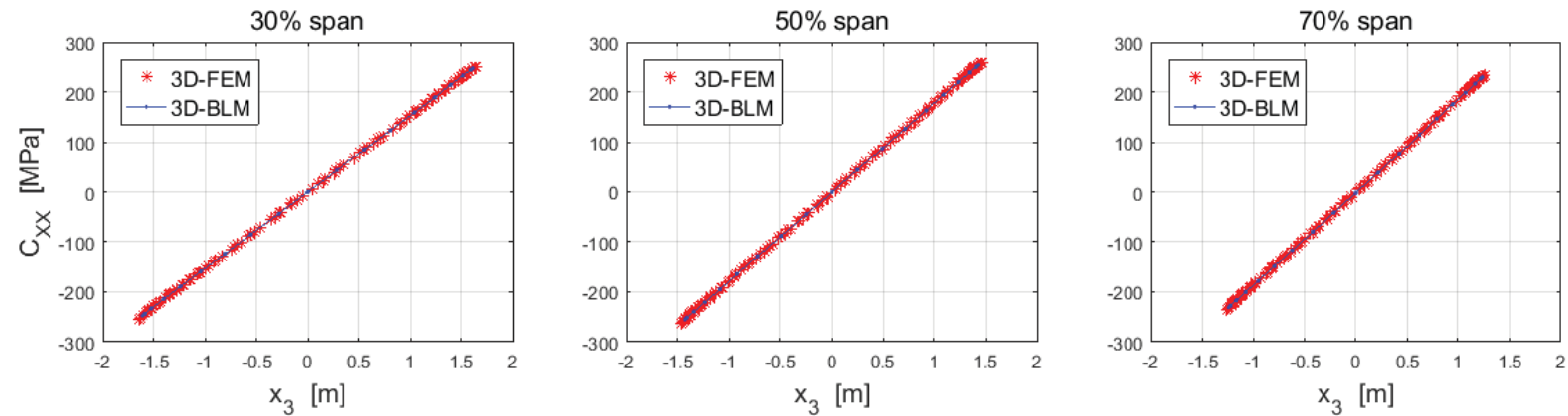

Figure 15: Stress field $\mathrm{C}_{\mathrm{XX}}$ at different cross-sections $(30 \%, 50 \%, 70 \%$ span) for $\mathrm{F}=5000 \mathrm{kN}$
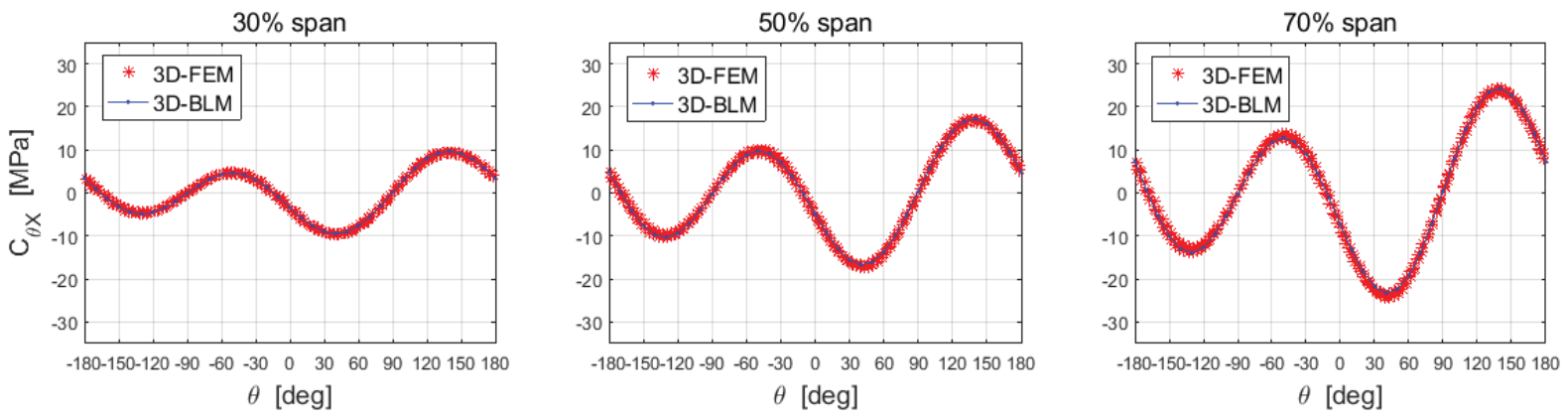

Figure 16: Stress field $\mathrm{C}_{\theta \mathrm{X}}$ at different cross-sections ( $30 \%, 50 \%, 70 \%$ span) for $\mathrm{F}=5000 \mathrm{kN}$
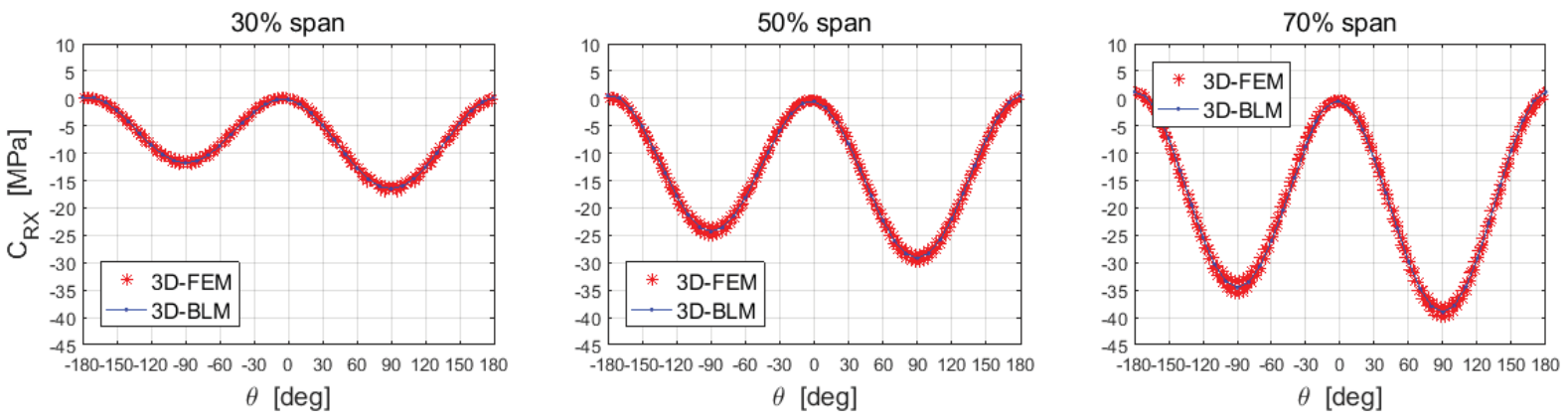

Figure 17: Stress field $\mathrm{C}_{\mathrm{RX}}$ at different cross-sections $(30 \%, 50 \%, 70 \%$ span) for $\mathrm{F}=5000 \mathrm{kN}$

The results shown in this example, which are based on the formulas derived in section 3.2, confirm once again the effectiveness of our modeling approach in terms of computational efficiency and accuracy with respect to nonlinear 3D-FEM approaches. Other examples can also be found in previous works (e.g. $[6,8,21])$, which address other geometries and load cases. 


\section{CONCLUSIONS}

Non-prismatic beamlike bodies are characterized by non-uniform cross-sections, yielding stress distributions not predicable via prismatic beam theories and requiring non-prismatic beam models. This paper has illustrated a modeling approach for non-prismatic beams subject to large displacements, 3D cross-sectional warpings and small strains. Specifically, it provides a computationally efficient and accurate model that accounts for the main geometric features of such elements (e.g. taper) and provides the stress fields as linear combinations of 1D strain measures and geometric parameters, with the coefficients of such linear combinations to be computed only once for a given cross-sectional shape as solutions of PDEs, a fact which helps reducing the computational effort required to solve the 3D nonlinear problem.

Two test cases have been presented to illustrate how the model can be used and which results it can provide. Analytical results have also been obtained, have been included in the numerical version of the model (3D-BLM) and have been compared with the results of nonlinear 3D-FEM analyses, confirming the efficiency and accuracy of the approach. Apart from the most theoretical outcomes of the model, it can be particularly useful for the design and optimization of non-prismatic elements used in engineering: in particular, on the one hand the closed-form formulas obtainable from the model can help an engineer since the preliminary design tasks; on the other hand, the model implemented in a numerical code can be used for multi-objective optimization tasks thanks to its computational efficiency and accuracy.

The results presented in this paper in terms of stress and strain fields have addressed the cross-sectional out-of-plane deformations and the corresponding PDEs. Investigations about the in-plane deformations and their PDEs would also be important, along with an analytical study about the effects of other geometric features (e.g. centre-line curvature) and those of the material non-homogeneity and anisotropy. An overview of other geometries that admit analytical closed-form solutions would also be interesting, plus other numerical examples validating the proposed modeling approach against other geometries and load cases. All such topics will be addressed in subsequent works.

\section{REFERENCES}

[1] Goodier G.N. and Griffin D.S., Elastic bending of pretwisted bars, Int. J. Solids Structures, 5, 1231-1245, 1969.

[2] Krenk S., The torsion-extension coupling in pretwisted elastic beams, Int. J. Solids Structures, 19, 67-72, 1983.

[3] Rosen A., Theoretical and experimental investigation of the nonlinear torsion and extension of initially twisted bars, J. Applied Mechanics, 50, 321-326, 1983.

[4] Rosen A., Structural and dynamic behavior of pretwisted rods and beams, American Society of Mechanical Engineers, 44, 483-515, 1991.

[5] Kunz D.L., Survey and comparison of engineering beam theories for helicopter rotor blades, J. of Aircraft, 31, 473-479, 1994.

[6] Migliaccio G., Ruta G., The influence of an initial twisting on tapered beams undergoing large displacements, Meccanica, 1-15, https://doi.org/10.1007/s11012-021-01334-2, 2021.

[7] Buckney N., Pirrera A., et al., On the structural topology of wind turbine blades, ThinWalled Structures, 67, 144-154, 2013. 
[8] Migliaccio G., Ruta G., et al., Beamlike models for the analyses of curved, twisted and tapered horizontal-axis wind turbine (HAWT) blades undergoing large displacements, Wind Energy Science, 5, 685-698, https://doi.org/10.5194/wes-5-685-2020, 2020.

[9] Migliaccio G., and Ruta G., Rotor blades as curved, twisted, and tapered beam-like structures subjected to large deflections, Engineering Structures, 222, 111089, 2020.

[10] Balduzzi G., Hochreiner G., and Füssl J., Stress recovery from one dimensional models for tapered bi-symmetric thin-walled I beams: deficiencies in modern engineering tools and procedures, Thin-Walled Structures, 119, 934-945, 2017.

[11] Love A.E.H., A treatise on the mathematical theory of elasticity, 4th ed., Dover Publications, NY, 1944.

[12] Timoshenko S.P., Goodier J.N., Theory of elasticity, 2nd ed., McGraw-Hill, 1951.

[13] Sokolnikoff I.S., Mathematical theory of elasticity, 1st ed., McGraw-Hill Inc., 1946.

[14] Simo J.C., A finite strain beam formulation, the three-dimensional dynamic problem, part I, Computer methods in applied mechanics and engineering, 49, 55-70, 1985.

[15] Ibrahimbegovic A., On finite element implementation of geometrically nonlinear Reissner's beam theory: three-dimensional curved beam elements, Computer methods in applied mechanics and engineering, 122, 11-26, 1995.

[16] Berdichevsky V.L., On the energy of an elastic rod, J. Appl. Mathematics and Mechanics, 45, 518-529, 1981.

[17] Yu W., Hodges D.H., and Ho J.C., Variational asymptotic beam-sectional analysis - an updated version, Int. J. Engineering Science, 59, 40-64, 2012.

[18] Migliaccio G., Ruta G, et al., Curved and twisted beam models for aeroelastic analysis of wind turbine blades in large displacements, XXIV AIMETA conference 2019, Lecture notes in mechanical engineering, Springer, 2020.

[19] Pai P.F., Three kinematic representations for modelling of high flexible beams and their applications, Int. J. Solids and Structures, 48, 2764-2777, 2011.

[20] Ruta G., Pignataro M., Rizzi N., A direct one-dimensional beam model for the flexuraltorsional buckling of thin-walled beams, J. Mechanics of Materials and Structures, 1, 1479-1496, 2006.

[21] Migliaccio G., Non-prismatic beamlike structures with 3D cross-sectional warping, in 14th World Congress in Computational Mechanics (WCCM) \& ECCOMAS congress 2020, Paris, France, 11-15 Jan. 2021.

[22] Gurtin M.E., An introduction to continuum mechanics, Mathematics in Science and Engineering, 1st ed., Academic Press, 1981.

[23] Courant R., and Hilbert D., Methods of mathematical physics, 1st ed., Interscience Publisher, 1953.

[24] Madenci E., and Guven I., The finite element method and applications in engineering using Ansys, 2nd ed., Springer, 2015. 